\title{
Number of Bound States of Schrödinger Operators with Matrix-Valued Potentials
}

\author{
Dedicated to Jean-Claude Cortet, in appreciation of his contribution to \\ Letters in Mathematical Physics
}

\author{
RUPERT L. FRANK ${ }^{1}$, ELLIOTT H. LIEB ${ }^{2}$ and ROBERT SEIRINGER ${ }^{3}$ \\ ${ }^{1}$ Department of Mathematics, Princeton University, Washington Road, Princeton, \\ NJ 08544, USA. e-mail: rlfrank@math.princeton.edu \\ ${ }^{2}$ Departments of Mathematics and Physics, Princeton University, P. O. Box 708, Princeton, \\ NJ 08544, USA. e-mail: lieb@princeton.edu \\ ${ }^{3}$ Department of Physics, Princeton University, P. O. Box 708, Princeton, NJ 08544, USA. \\ e-mail: rseiring@princeton.edu
}

Received: 9 October 2007 / Revised: 15 November 2007 / Accepted: 16 November 2007 Published online: 5 December 2007

\begin{abstract}
We give a Cwikel-Lieb-Rozenblum type bound on the number of bound states of Schrödinger operators with matrix-valued potentials using the functional integral method of Lieb. This significantly improves the constant in this inequality obtained earlier by Hundertmark.
\end{abstract}

Mathematics Subject Classification (2000). Primary: 35P15; Secondary: 35J10, 47F05, 81Q10.

Keywords. Schrödinger operator, Lieb-Thirring inequality, Cwikel-Lieb-Rozenblum inequality.

\section{Introduction}

We consider the Schrödinger operator $-\Delta-V(x)$ on $\mathbb{R}^{d}$, but with the difference from the usual case that $V$ is a Hermitian matrix-valued potential. In other words, the Hilbert space is not $L^{2}\left(\mathbb{R}^{d}\right)$ but $L^{2}\left(\mathbb{R}^{d} ; \mathbb{C}^{N}\right)$. The values of functions in this space, $\psi(x)$, are $N$-dimensional vectors. (What we say here easily generalizes to "operator-valued" potentials, i.e., $\mathbb{C}^{N}$ is replaced by a Hilbert space such as $L^{2}\left(\mathbb{R}^{m}\right)$, but we stay with matrices in order to avoid technicalities.) The Cwikel-Lieb-Rozenblum (CLR) bound for $d \geq 3$ in the scalar case $N=1$ states that $\#(-\Delta-V)$, the number of negative eigenvalues of $-\Delta-V$, can be estimated by

$$
\#(-\Delta-V) \leq L_{0, d} \int_{\mathbb{R}^{d}} V_{+}(x)^{d / 2} \mathrm{~d} x .
$$

(C) 2007 by the authors. This paper may be reproduced, in its entirety, for non-commercial purposes.

This work was supported by DAAD grant D/06/49117 (R.F.), by U.S. National Science Foundation grants PHY 0652854 (E.L.) and PHY 0652356 (R.S.), and by an A.P. Sloan Fellowship (R.S.). 
(Here and below $v_{ \pm}:=(|v| \pm v) / 2$ denotes the positive and negative part of $v$.) We remind the reader that the "semi-classical" approximation to $\#(-\Delta-V)$ is given in the scalar case by the phase space volume

$$
(2 \pi)^{-d} \underset{\left\{(p, x) \in \mathbb{R}^{d} \times \mathbb{R}^{d}: p^{2}-V(x)<0\right\}}{\int} \mathrm{d} p \mathrm{~d} x=L_{0, d}^{\mathrm{cl}} \int_{\mathbb{R}^{d}} V_{+}(x)^{d / 2} \mathrm{~d} x,
$$

where

$$
L_{0, d}^{\mathrm{cl}}=(2 \pi)^{-d} \int_{\left\{p \in \mathbb{R}^{d}: p^{2}<1\right\}} \mathrm{d} p=\left(2^{d} \pi^{d / 2} \Gamma(d / 2+1)\right)^{-1} .
$$

The bound (1.1) was obtained by completely independent methods in $[3,14,15,18$, 19]. Later, different proofs were given in $[2,13]$. The best constant, which is close to optimal for $d=3$, was obtained in $[14,15]$ using the Feynman-Kac formula and Jensen's inequality.

Our goal here is to extend inequality (1.1) to the matrix case (with a possibly different constant $\left.L_{0, d}\right)$. The motivation for this extension was the work of Laptev and Weidl [11] (see also [10]) who realized that the extension allowed one to conclude that good/sharp constants obtained in low dimensions would automatically give good/sharp constants in higher dimensions. The fact that the inequality (1.1) is valid in the matrix case was proved by Hundertmark [6], confirming a conjecture in [12]. He follows Cwikel's method and obtains a constant which is far from optimal. Hundertmark points out that "it would be nice to extend Lieb's [...] proof of the CLR-bound to operator-valued potentials". This is the content of this letter.

THEOREM 1.1. Let $d \geq 3$ and assume that $V$ is a function on $\mathbb{R}^{d}$ taking values in the Hermitian $N \times N$ matrices. Then

$$
\#(-\Delta-V) \leq R_{0, d} L_{0, d}^{\mathrm{cl}} \int_{\mathbb{R}^{d}} \operatorname{Tr}_{\mathbb{C}^{N}}\left[V_{+}(x)^{d / 2}\right] \mathrm{d} x,
$$

where $R_{0, d} \leq 10.332$ and $V_{+}:=(|V|+V) / 2$.

The constant 10.332 will be obtained for $d=3$ and, by the Laptev-Weidl method (as used by Hundertmark [6]) it is valid uniformly for all $d \geq 3$. We emphasize that our bound on $R_{0, d}$ is slightly worse than the constant 6.87 in [14,15] for the scalar case $N=1$. Still, it improves that of [6] by almost one order of magnitude. For $d=3$ our bound on $R_{0,3}$ is at most a factor 2.24 bigger than the optimal constant in (1.2), since it is known that $R_{0,3} \geq 8 / \sqrt{3} \approx 4.619$ [16]. 
It is well known that by a simple integration the bound (1.2) yields the Lieb-Thirring inequalities

$$
\operatorname{Tr}_{L_{2}\left(\mathbb{R}^{d} ; \mathbb{C}^{N}\right)}(-\Delta-V)_{-}^{\gamma} \leq R_{\gamma, d} L_{\gamma, d}^{\mathrm{cl}} \int_{\mathbb{R}^{d}} \operatorname{Tr}_{\mathbb{C}^{N}}\left[V_{+}(x)^{\gamma+d / 2}\right] \mathrm{d} x
$$

for all $\gamma>0, d \geq 3$ with $R_{\gamma, d} \leq R_{0, d} \leq 10.332$ and

$$
L_{\gamma, d}^{\mathrm{cl}}=(2 \pi)^{-d} \int_{\mathbb{R}^{d}}\left(1-p^{2}\right)_{+}^{\gamma} \mathrm{d} p
$$

Indeed, $R_{\gamma, d}$ is a monotone non-increasing function of $\gamma$ [1]. Even in the scalar case $N=1$, this yields the best known constants in this inequality for the parameter range $0<\gamma<1 / 2$. For comparison we recall that the best known bounds for larger values of $\gamma$ are $R_{\gamma, d} \leq 2 \pi / \sqrt{3} \approx 3.628$ if $\gamma \geq 1 / 2$ and $R_{\gamma, d} \leq \pi / \sqrt{3} \approx 1.814$ if $\gamma \geq 1[4,8]$. For $\gamma \geq 3 / 2$ one has $R_{\gamma, d}=1$, which is sharp [11]. We refer to the surveys [7,12] for more about inequalities (1.3).

Apart from yielding very accurate constants we believe that there is a mathematical interest in extending the path-integral method in $[14,15]$ to the operator-valued situation. In contrast to the method of [3] used in [6], which is rather rigidly based on mapping properties of the Fourier transform, the method of [14, 15] used here works in much wider generality, e.g., on Riemannian manifolds. The only input needed is an upper bound on the heat kernel of the (scalar) unperturbed operator. For example, the Hardy-Lieb-Thirring bounds in [5] extend to the matrix-valued situation.

As already pointed out, we proceed similarly to $[14,15]$. Therefore we will be brief at some points and ignore some technicalities. There is an important new ingredient in our proof, however. Since matrices $W_{1}, \ldots, W_{n}$ do not commute, in general, we need to work with the "time ordering" of a function $f\left(\sum_{j} W_{j}\right)$ of their sum. In Proposition 3.1 we shall prove a modification of Jensen's inequality valid in this setting for a certain class of convex functions $f$.

\section{A Trace Formula}

Given self-adjoint $N \times N$-matrices $W_{1}, \ldots, W_{n}$ and a function $f$ on $\mathbb{R}$, the matrix $f\left(\sum_{j} W_{j}\right)$ is defined by the spectral projections of $\sum_{j} W_{j}$. Instead, we introduce the "time-ordering" of the matrix $f\left(\sum_{j} W_{j}\right)$ as follows. We write $W_{j}$ in its spectral representation

$$
W_{j}=\sum_{k=1}^{N} w_{k}^{(j)} P_{k}^{(j)}
$$


where $w_{k}^{(j)}$ are the eigenvalues and $P_{k}^{(j)}$ the corresponding orthogonal projections, and define

$$
\mathcal{T} f\left(W_{1}, \ldots, W_{n}\right):=\sum_{k_{1}, \ldots, k_{n}=1}^{N} f\left(\sum_{j=1}^{n} w_{k_{l}}^{(j)}\right) P_{k_{1}}^{(1)} \ldots P_{k_{n}}^{(n)} .
$$

Intuitively, this means that when calculating $f\left(\sum_{j} W_{j}\right)$, one puts all the $W_{1}$ 's left of the $W_{2}$ 's, the $W_{2}$ 's left of the $W_{3}$ 's, and so on, without worrying about commutators. It is instructive to look at some examples.

EXAMPLE 2.1. If $f(\mu)=\mu^{k}, k \in \mathbb{N}$, then the definition immediately implies

$$
\mathcal{T} f\left(W_{1}, \ldots, W_{n}\right)=\sum_{j_{1}+\cdots+j_{n}=k} \frac{k !}{j_{1} ! \ldots j_{n} !} W_{1}^{j_{1}} \ldots W_{n}^{j_{n}} .
$$

EXAMPLE 2.2. If $f(\mu)=\mathrm{e}^{\alpha \mu}, \alpha \in \mathbb{R}$, then again by the definition (2.1)

$$
\mathcal{T} f\left(W_{1}, \ldots, W_{n}\right)=\mathrm{e}^{\alpha W_{1}} \ldots \mathrm{e}^{\alpha W_{n}} .
$$

Similarly, one shows that if $f(\mu)=\mu \mathrm{e}^{\alpha \mu}, \alpha \in \mathbb{R}$, then

$$
\begin{aligned}
& \mathcal{T} f\left(W_{1}, \ldots, W_{n}\right)= \\
& \quad=W_{1} \mathrm{e}^{\alpha W_{1}} \mathrm{e}^{\alpha W_{2}} \ldots \mathrm{e}^{\alpha W_{n}}+\mathrm{e}^{\alpha W_{1}} W_{2} \mathrm{e}^{\alpha W_{2}} \ldots \mathrm{e}^{\alpha W_{n}}+\cdots+\mathrm{e}^{\alpha W_{1}} \mathrm{e}^{\alpha W_{2}} \ldots W_{n} \mathrm{e}^{\alpha W_{n}} .
\end{aligned}
$$

We have introduced the notion of time-ordering in order to generalize the trace formula in $[14,15]$, which is the starting point of the analysis leading to (1.1).

PROPOSITION 2.3. Let $f$ be a non-negative, lower semi-continuous function $f$ with $f(0)=0$, and let

$$
F(\lambda):=\int_{0}^{\infty} f(\mu) \mathrm{e}^{-\mu / \lambda} \mu^{-1} \mathrm{~d} \mu, \quad \lambda>0 .
$$

Then for any sufficiently regular and decaying functions $V$ on $\mathbb{R}^{d}, d \geq 3$, taking values in the non-negative $N \times N$-matrices, one has

$$
\begin{aligned}
& \operatorname{Tr}_{L_{2}\left(\mathbb{R}^{d} ; \mathbb{C}^{N}\right)} F\left(V^{1 / 2}(-\Delta)^{-1} V^{1 / 2}\right)= \\
& =\int_{0}^{\infty} \frac{\mathrm{d} t}{t} \lim _{n \rightarrow \infty} \int_{\mathbb{R}^{d}} \cdots \int_{\mathbb{R}^{d}} \mathrm{~d} x_{1} \cdots \mathrm{d} x_{n} \times \\
& \quad \times \prod_{j=1}^{n} k\left(x_{j}, x_{j-1}, \frac{t}{n}\right) \operatorname{Tr}_{\mathbb{C}^{N}}\left[\mathcal{T} f\left(\frac{t}{n} V\left(x_{1}\right), \ldots, \frac{t}{n} V\left(x_{n}\right)\right)\right]
\end{aligned}
$$

with the convention that $x_{0}=x_{n}$. Here, $k(x, y, t)=(4 \pi t)^{-d / 2} \exp \left(-|x-y|^{2} /(4 t)\right)$. 
In the limit $n \rightarrow \infty$ the multiple integral on the right side of (2.3) converges to a Wiener integral (the Feynman-Kac integral); in fact, the right side of (2.3) is the Trotter product approximation to this integral $[9,17,21]$.

Proof. By an approximation argument [21, Theorem 8.2] it suffices to prove this formula for

$$
F(\lambda)=\lambda /(1+\alpha \lambda), \quad f(\mu)=\mu \mathrm{e}^{-\alpha \mu},
$$

where $\alpha>0$ is a constant. Using the resolvent identity and Trotter's product formula, one easily verifies that in this case

$$
\begin{aligned}
F\left(V^{1 / 2}(-\Delta)^{-1} V^{1 / 2}\right) & =V^{1 / 2}(-\Delta+\alpha V)^{-1} V^{1 / 2}= \\
& =\int_{0}^{\infty} V^{1 / 2} \exp (-t(-\Delta+\alpha V)) V^{1 / 2} \mathrm{~d} t= \\
& =\int_{0}^{\infty} \lim _{n \rightarrow \infty} T_{n}(t) \mathrm{d} t .
\end{aligned}
$$

Here,

$$
T_{n}(t):=V^{1 / 2}(\exp (t \Delta / n) \exp (-t \alpha V / n))^{n} V^{1 / 2} .
$$

The latter is an integral operator and we evaluate its trace by integrating its kernel on the diagonal. Let $k$ denote the heat kernel

$$
k(x, y, t):=(4 \pi t)^{-d / 2} \exp \left(-|x-y|^{2} /(4 t)\right) .
$$

Then

$$
\begin{aligned}
& \operatorname{Tr}_{L_{2}\left(\mathbb{R}^{d} ; \mathbb{C}^{N}\right)} T_{n}(t)= \\
& \quad=\int \ldots \int \mathrm{d} x_{1} \ldots \mathrm{d} x_{n} \prod_{j=1}^{n} k\left(x_{j}, x_{j-1}, \frac{t}{n}\right) \operatorname{Tr}_{\mathbb{C}^{N}}\left[\mathrm{e}^{-\frac{\alpha t}{n} V\left(x_{1}\right)} \ldots \mathrm{e}^{-\frac{\alpha t}{n} V\left(x_{n}\right)} V\left(x_{n}\right)\right] .
\end{aligned}
$$

Cyclical relabeling of the variables leads to

$$
\begin{aligned}
& \operatorname{Tr}_{L_{2}\left(\mathbb{R}^{d} ; \mathbb{C}^{N}\right)} T_{n}(t)= \\
& \quad=\frac{1}{t} \int \ldots \int \mathrm{d} x_{1} \ldots \mathrm{d} x_{n} \prod_{j=1}^{n} k\left(x_{j}, x_{j-1}, \frac{t}{n}\right) \operatorname{Tr}_{\mathbb{C}^{N}}\left[\mathcal{T} f\left(t V\left(x_{1}\right) / n, \ldots, t V\left(x_{n}\right) / n\right)\right]
\end{aligned}
$$

(compare with Example 2.2). The claimed formula (2.3) follows if one interchanges the trace with the $t$-integration and the $n$-limit. 


\section{Jensen's Inequality and Time Ordering}

To apply (2.3) we need to estimate the trace of a time-ordered sum. Recall that Jensen's inequality says that $\operatorname{Tr} f\left(\sum W_{j}\right) \leq n^{-1} \sum \operatorname{Tr} f\left(n W_{j}\right)$ for $f$ convex. The analog for the time-ordered case, and a certain class of $f$ 's, is

PROPOSITION 3.1. Assume that

$$
f(\mu)=\sum_{j=0}^{\infty} \alpha_{j} \mu^{j}+\int_{\mathbb{R}} \mathrm{e}^{-\alpha \mu} \mathrm{d} v(\alpha)
$$

for some $\alpha_{0}, \alpha_{1} \in \mathbb{R}, \alpha_{j} \geq 0$ for $j \geq 2$ and a non-negative measure $v$. Then for any non-negative $N \times N$-matrices $W_{1}, \ldots, W_{n}$

$$
\operatorname{Re} \operatorname{Tr}_{\mathbb{C}^{N}}\left[\mathcal{T} f\left(W_{1}, \ldots, W_{n}\right)\right] \leq \frac{1}{n} \sum_{j=1}^{n} \operatorname{Tr}_{\mathbb{C}^{N}} f\left(n W_{j}\right) .
$$

Note that the $f$ in (3.1) is convex on $[0, \infty)$. We do not know whether the statement is true for an arbitrary convex function on $[0, \infty)$. If it were, the constant in Theorem 1.1 could be improved, as explained at the end of this letter.

Proof. By linearity of the trace it suffices to consider the cases $f(\mu)=\mu^{k}, k \in \mathbb{N}$, and $f(\mu)=\mathrm{e}^{\alpha \mu}$. In the former case, one has by Hölder's inequality for traces (see, e.g., $[20$, Theorem 2.8])

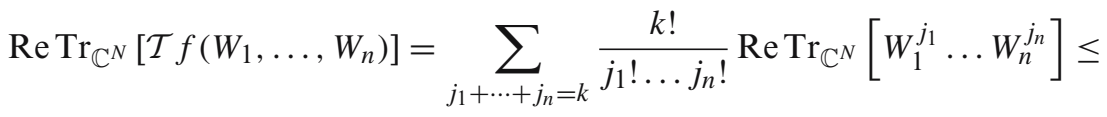

$$
\begin{aligned}
& \leq \sum_{j_{1}+\cdots+j_{n}=k} \frac{k !}{j_{1} ! \ldots j_{n} !}\left(\operatorname{Tr}_{\mathbb{C}^{N}} W_{1}^{k}\right)^{j_{1} / k} \ldots\left(\operatorname{Tr}_{\mathbb{C}^{N}} W_{n}^{k}\right)^{j_{n} / k}= \\
& =f\left(\sum_{j=1}^{n}\left(\operatorname{Tr}_{\mathbb{C}^{N}} W_{j}^{k}\right)^{1 / k}\right),
\end{aligned}
$$

and the assertion follows from the convexity of $f$. In the latter case, one has similarly by Hölder's inequality and the geometric-arithmetic mean inequality

$$
\begin{aligned}
& \operatorname{Re} \operatorname{Tr}_{\mathbb{C}^{N}}\left[\mathcal{T} f\left(W_{1}, \ldots, W_{n}\right)\right]=\operatorname{Re} \operatorname{Tr}_{\mathbb{C}^{N}}\left[\mathrm{e}^{\alpha W_{1}} \ldots \mathrm{e}^{\alpha W_{n}}\right] \leq \\
& \leq\left(\operatorname{Tr}_{\mathbb{C}^{N}} \mathrm{e}^{\alpha n W_{1}}\right)^{1 / n} \cdots\left(\operatorname{Tr}_{\mathbb{C}^{N}} \mathrm{e}^{\alpha n W_{n}}\right)^{1 / n} \leq \\
& \leq \frac{1}{n} \sum_{j=1}^{n} \operatorname{Tr}_{\mathbb{C}^{N}} \mathrm{e}^{n \alpha W_{j}}
\end{aligned}
$$

as claimed. 
COROLLARY 3.2. Assume that $f$ is a non-negative function of the form considered in Proposition 3.1 and let $F$ be as in (2.2). Then for any sufficiently regular and decaying function $V$ on $\mathbb{R}^{d}$ taking values in the non-negative $N \times N$-matrices, one has

$$
\begin{aligned}
& \operatorname{Tr}_{L_{2}\left(\mathbb{R}^{d} ; \mathbb{C}^{N}\right)} F\left(V^{1 / 2}(-\Delta)^{-1} V^{1 / 2}\right) \leq \\
& \quad \leq \frac{1}{(4 \pi)^{d / 2}}\left(\int_{0}^{\infty} \frac{f(s)}{s^{d / 2}} \frac{\mathrm{d} s}{s}\right) \int_{\mathbb{R}^{d}} \operatorname{Tr}_{\mathbb{C}^{N}}\left[V(x)^{d / 2}\right] \mathrm{d} x .
\end{aligned}
$$

Proof. Combining Propositions 3.1 and 2.3 we obtain

$$
\begin{aligned}
& \operatorname{Tr}_{L_{2}\left(\mathbb{R}^{d} ; \mathbb{C}^{N}\right)} F\left(V^{1 / 2}(-\Delta)^{-1} V^{1 / 2}\right) \leq \\
& \quad \leq \int_{0}^{\infty} \frac{\mathrm{d} t}{t} \lim _{n \rightarrow \infty} \int_{\mathbb{R}^{d}} \ldots \int_{\mathbb{R}^{d}} \prod_{j=1}^{n} k\left(x_{j}, x_{j-1}, \frac{t}{n}\right) \frac{1}{n} \sum_{j=1}^{n} \operatorname{Tr}_{\mathbb{C}^{N}} f\left(t V\left(x_{j}\right)\right) \mathrm{d} x_{1} \ldots \mathrm{d} x_{n} .
\end{aligned}
$$

(Here we have used that the left side of (2.3) is real, hence only the real part of $\operatorname{Tr} \mathcal{T} f$ contributes to the integral.) The semi-group property implies

$$
\begin{aligned}
& \frac{1}{n} \int_{\mathbb{R}^{d}} \ldots \int_{\mathbb{R}^{d}} \prod_{j=1}^{n} k\left(x_{j}, x_{j-1}, \frac{t}{n}\right) \sum_{j=1}^{n} \operatorname{Tr}_{\mathbb{C}^{N}} f\left(t V\left(x_{j}\right)\right) \mathrm{d} x_{1} \ldots \mathrm{d} x_{n}= \\
& \quad=\frac{1}{n} \sum_{j=1}^{n} \int_{\mathbb{R}^{d}} k\left(x_{j}, x_{j}, t\right) \operatorname{Tr}_{\mathbb{C}^{N}} f\left(t V\left(x_{j}\right)\right) \mathrm{d} x_{j}=\frac{1}{(4 \pi t)^{d / 2}} \int_{\mathbb{R}^{d}} \operatorname{Tr}_{\mathbb{C}^{N}} f(t V(x)) \mathrm{d} x .
\end{aligned}
$$

Denoting the eigenvalues of $V(x)$ by $v_{1}(x) \leq \cdots \leq v_{N}(x)$ one finds that

$$
\int_{0}^{\infty} \frac{\mathrm{d} t}{t} \frac{\operatorname{Tr}_{\mathbb{C}^{N}} f(t V(x))}{t^{d / 2}}=\sum_{j=1}^{N} \int_{0}^{\infty} \frac{\mathrm{d} t}{t} \frac{f\left(t v_{j}(x)\right)}{t^{d / 2}}=\sum_{j=1}^{N} v_{j}(x)^{d / 2} \int_{0}^{\infty} \frac{\mathrm{d} s}{s} \frac{f(s)}{s^{d / 2}},
$$

thereby proving the assertion.

\section{Proof of Theorem 1.1}

First we assume that $d=3$. By the variational principle we can assume that $V(x)$ is a non-negative matrix for all $x$, and by an approximation argument we can assume that $V$ is smooth and rapidly decaying. For any increasing function $F$ on $(0, \infty)$ the Birman-Schwinger principle implies that

$$
\#(-\Delta-V) \leq F(1)^{-1} \operatorname{Tr}_{L^{2}\left(\mathbb{R}^{3} ; \mathbb{C}^{N}\right)} F\left(V^{1 / 2}(-\Delta)^{-1} V^{1 / 2}\right) .
$$


We choose $F=F_{a}$ of the form (2.2) where $a>0$ is a parameter and $f=f_{a}$ is defined by

$$
f_{a}(\mu)=\frac{\mu^{2}}{\mu+a}=\mu-a+\frac{a^{2}}{\mu+a}=\mu-a+a^{2} \int_{0}^{\infty} \mathrm{e}^{-t(\mu+a)} \mathrm{d} t .
$$

Since this function is of the form considered in Proposition 3.1 we can apply Corollary 3.2 and get in view of (4.1)

$$
\#(-\Delta-V) \leq C_{a} \int_{\mathbb{R}^{3}} \operatorname{Tr}_{\mathbb{C}^{N}}\left[V(x)^{3 / 2}\right] \mathrm{d} x,
$$

where

$$
\begin{aligned}
C_{a} & :=(4 \pi)^{-3 / 2} F_{a}(1)^{-1}\left(\int_{0}^{\infty} \frac{f_{a}(s)}{s^{3 / 2}} \frac{\mathrm{d} s}{s}\right) \\
& =\frac{1}{8}(\pi a)^{-1 / 2}\left(1+a \mathrm{e}^{a} \int_{a}^{\infty} \mathrm{e}^{-s} \frac{\mathrm{d} s}{s}\right)^{-1} .
\end{aligned}
$$

The result follows by choosing $a=1.13$, which approximately minimizes $C_{a}$.

Now we assume that $d \geq 4$. We will use the Laptev-Weidl strategy to reduce this case to the case $d=3$. This argument is already contained in [6] but we include it for the sake of completeness. We note that by a straightforward approximation argument as in [11] the inequality for $d=3$ holds also for $N=\infty$, i.e., if $V(x)$ assumes values in the compact self-adjoint operators on a separable Hilbert space. Introduce variables $x=\left(x_{1}, x_{2}\right) \in \mathbb{R}^{d}$ where $x_{1} \in \mathbb{R}^{3}$ and $x_{2} \in \mathbb{R}^{d-3}$. We decompose the Laplacian correspondingly as $-\Delta=-\Delta_{1}-\Delta_{2}$ and define, for fixed $x_{1} \in$ $\mathbb{R}^{3}, W\left(x_{1}\right):=\left(-\Delta_{1}-V\left(x_{1}, \cdot\right)\right)_{-}$. If $V$ is, say, smooth with compact support, then $W\left(x_{1}\right)$ is a compact operator in $L^{2}\left(\mathbb{R}^{d-3}, \mathbb{C}^{N}\right)$ for every $x_{1}$. The variational principle and the inequality for $d=3$ imply that

$$
\#(-\Delta-V) \leq \#\left(-\Delta_{1}-W\right) \leq R_{0,3} L_{0,3}^{\mathrm{cl}} \int_{\mathbb{R}^{3}} \operatorname{Tr}_{L^{2}\left(\mathbb{R}^{d-3}, \mathbb{C}^{N}\right)}\left[W\left(x_{1}\right)^{3 / 2}\right] \mathrm{d} x_{1} .
$$

By the result of Laptev and Weidl [11], one has

$$
\operatorname{Tr}_{L^{2}\left(\mathbb{R}^{d-3}, \mathbb{C}^{N}\right)}\left[W\left(x_{1}\right)^{3 / 2}\right] \leq L_{3 / 2, d-3}^{\mathrm{cl}} \int_{\mathbb{R}^{d-3}} \operatorname{Tr}_{\mathbb{C}^{N}}\left[V\left(x_{1}, x_{2}\right)^{d / 2}\right] \mathrm{d} x_{2}
$$

with the constant $L_{3 / 2, d-3}^{\mathrm{cl}}$ from (1.4). Noting that $L_{0,3}^{\mathrm{cl}} L_{3 / 2, d-3}^{\mathrm{cl}}=L_{0, d}^{\mathrm{cl}}$ we obtain the assertion of Theorem 1.1. 
Remark 4.1. If the estimate in Proposition 3.1 held for all convex functions on $[0, \infty)$ [not merely for those of the form (3.1)], then we could choose $f_{a}(\mu)=$ $(\mu-a)_{+}$in the preceding proof, as in [14,15], and would get the same constant as in the scalar case. If estimate (3.2) held for the absolute value instead of merely for the real part, our proof would extend to Schrödinger operators with magnetic fields. This follows as in the scalar case by means of the diamagnetic inequality.

\section{References}

1. Aizenman, M., Lieb, E.H.: On semi-classical bounds for eigenvalues of Schrödinger operators. Phys. Lett. 66, 427-429 (1978)

2. Conlon, J.G.: A new proof of the Cwikel-Lieb-Rosenbljum bound. Rocky Mt. J. Math. 15(1), 117-122 (1985)

3. Cwikel, M.: Weak type estimates for singular values and the number of bound states of Schrödinger operators. Ann. Math. 106, 93-102 (1977)

4. Dolbeault, J., Laptev, A., Loss, M.: Lieb-Thirring inequalities with improved constants. J. Eur. Math. Soc. (to appear) Preprint: arXiv:0708.1165v2 [math.AP]

5. Frank, R.L., Lieb, E.H., Seiringer, R.: Hardy-Lieb-Thirring inequalities for fractional Schrödinger operators. J. Am. Math. Soc. (to appear) Preprint: arXiv:math/0610593v2 [math.SP]

6. Hundertmark, D.: On the number of bound states for Schrödinger operators with operator-valued potentials. Ark. Mat. 40, 73-87 (2002)

7. Hundertmark, D.: Some bound state problems in quantum mechanics. Spectral theory and mathematical physics: a Festschrift in honor of Barry Simon's 60th birthday, pp. 463-496, Proc. Sympos. Pure Math. vol. 76. Part 1. American Mathematical Societys, Providence (2007)

8. Hundertmark, D., Laptev, A., Weidl, T.: New bounds on the Lieb-Thirring constants. Invent. Math. 40, 693-704 (2000)

9. Ichinose, T.: Norm convergence of the Trotter product formula for Schrödinger operators via the Feynman-Kac formula. In: Path integrals: Dubna '96, pp. 341-346, Joint Inst. Nuclear Res., Dubna (1996)

10. Laptev, A.: Dirichlet and Neumann eigenvalue problems on domains in Euclidean spaces. J. Funct. Anal. 151(2), 531-545 (1997)

11. Laptev, A., Weidl, T.: Sharp Lieb-Thirring inequalities in high dimensions. Acta Math. 184, 87-111 (2000)

12. Laptev, A., Weidl, T.: Recent results on Lieb-Thirring inequalities. Journées 'Équations aux Dérivées Partielles' (La Chapelle sur Erdre, 2000), Exp. No. XX, Univ. Nantes, Nantes (2000) http://www.numdam.org/numdam-bin/fitem?id=JEDP_2000____A20_0

13. Li, P., Yau, S.T.: On the Schrödinger equation and the eigenvalue problem. Commun. Math. Phys. 88(3), 309-318 (1983)

14. Lieb, E.H.: Bounds on the eigenvalues of the Laplace and Schrödinger operators. Bull. Amer. Math. Soc. 82, 751-752 (1976)

15. Lieb, E.H.: The number of bound states of one body Schrödinger operators and the Weyl problem. Proc. A.M.S. Symp. Pure Math. 36, 241-252 (1980)

16. Lieb, E.H., Thirring, W.: Inequalities for the moments of the eigenvalues of the Schrödinger Hamiltonian and their relation to Sobolev inequalities. Stud. Math. Phys., pp. 269-303. Princeton University Press, Princeton (1976) 
17. Rozenblum, G.V., Solomyak, M.: The Cwikel-Lieb-Rozenblyum estimator for generators of positive semigroups and semigroups dominated by positive semigroups. St. Petersburg Math. J. 9(6), 1195-1211 (1998)

18. Rozenblum, G.V.: Distribution of the discrete spectrum of singular differential operators. Soviet Math. Dokl. 13, 245-249 (1972)

19. Rozenblum, G.V.: Distribution of the discrete spectrum of singular differential operators. Soviet Math. (Iz. VUZ) 20, 63-71 (1976)

20. Simon, B.: Trace ideals and their applications, 2nd edn. Mathematical Surveys and Monographs, vol. 120. American Mathematical Society, Providence (2005)

21. Simon, B.: Functional Integration and Quantum Physics, 2nd edn. American Mathematical Society, Providence (2005) 